\title{
Sweet fifteen
}

As Nature Physics turns fifteen, we celebrate some of our favourite papers.

$\mathrm{H}$ ow quickly times change. The last time we looked back at our previous achievements, in 2015 , we were ten ${ }^{1}$. We will readily admit that nothing could quite have prepared us for the turbulent five years that we have experienced since: Brexit, Trump and the COVID-19 pandemic, to name but three prominent developments on the world stage, have all tested our naive faith in the inexorable development of our collective wellbeing.

The polarizing emotions surrounding these events have made us aware of a whole new facet of the human condition; one that, as the expert rationalizers that we are, we initially struggled to identify and come to terms with. In short: we are now teenagers, and life is more complicated than we thought.

That being said, during this time we have also been lucky to witness a number of memorable scientific discoveries. LIGO's observation of gravitational waves, the first image of a black hole obtained by the Event Horizon Telescope Collaboration, and the discovery of superconductivity in bilayer graphene would all be worthy additions to Jorge Cham's 'Top 10 physics discoveries of the past decade ${ }^{2}$. It remains an exciting time as any to be a physicist.

And it is with this spirit that we have decided to mark the 15-year anniversary of Nature Physics. All the editors of the journal - past and present - have weighed in with their thoughts in a Feature highlighting their favourite papers from our back catalogue.

Our selection naturally reflects the breadth of our different interests as editors: for example, our launching editor, Alison Wright, focuses on our so-called front half, which under her tenure came to be a vibrant venue covering all manner of topics at the intersection between physics, art and society. Our newest recruit Richard Brierley, on the other hand, reminisces about a quantum simulation paper that inspired him as a young graduate student.

Other results that we dwell on admiringly include a 15-minute-long confinement of
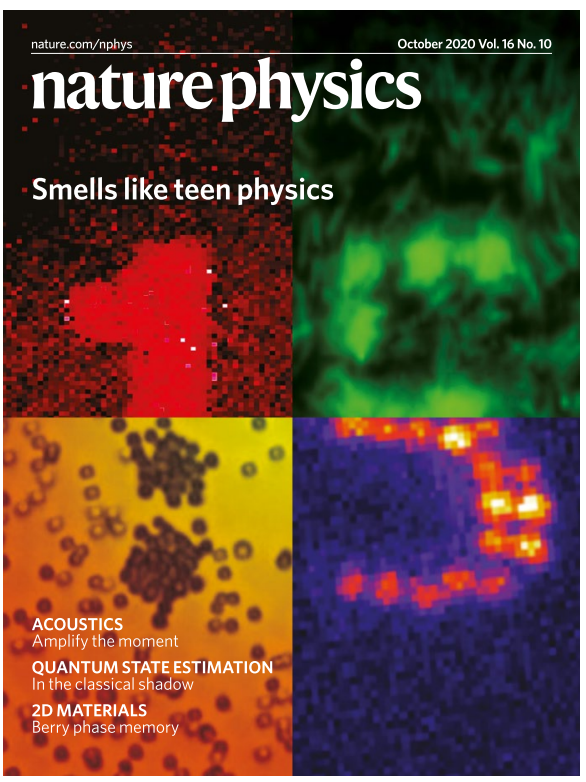

Our 15-year anniversary cover contains (clockwise from top left): image created by quantum imaging, self-assembly of green-fluorescent-protein-active Escherichia coli bacteria, a quantum gas microscopy image, and self-assembly of polystyrene beads. Credit: Hugo Defienne and Daniele Faccio, University of Glasgow (top left); Serim Ilday, Bilkent University - UNAM (top right and bottom left); Immanuel Bloch, Max Planck Institute of Quantum Optics (bottom right)

antihydrogen, electrons arranged in a fractal structure, and a laboratory model recreating a Parker spiral - the spiralling magnetic structure arising from the interaction between the Sun's magnetic field and the solar wind.

We celebrate these results not only for the beautiful phenomena that they uncover, but also - and this is in keeping with over 150 years of tradition at Nature - for the incredible feats of human ingenuity that made them observable in the first place. Indeed, if there is one recurring theme in the Feature (over and above physics, that is), it is the human touch that each one of us seems keen to share.
Every discovery has an interesting backstory. It might be the serendipitous nature of how it came about, or it might be the moment of realization that comes when seeing a plot, listening to a talk or just talking with somebody in the corridor every scientist likes to recount their own. This human side of the research endeavour rarely makes it into the actual papers we publish and, arguably, it is even rarer to hear the editors' side of the story: what did they ${ }^{*}$ really* think of the manuscript? What really piqued their interest?

As the contributions in the Feature hopefully show, editors are ultimately driven by a wish to champion the fields and communities at the journal, and to showcase them as widely as possible. We really aren't objective enough to be able to meaningfully claim this approach works - of course we think it does, but ultimately it's up to the readers to decide. But there is also a more subtle consequence to all this: the choices that we make inevitably mean that the editors' personality also comes through. And as a result, Nature Physics has a distinct personality of its own. In these days of increasing calls for everything to be data-driven, it is perhaps anathema to admit this, but there we are.

Five years ago, we wrote that "As long as it will be humans that practice science, its narrative will continue to be important. We will therefore remain focused on being a venue for the communication of the most important physics developments of the day, while at the same time fostering the appreciation that these great works of science deserve beyond their specialist communities". The physics may be changing, as is the world around us. But our mission and our enthusiasm for it remain undimmed.

Published online: 1 October 2020 https://doi.org/10.1038/s41567-020-01068-0

References

1. Nat. Phys. 11, 789-790 (2015).

2. Cham, J. Nat. Phys. 11, 799 (2015). 